\title{
Mechanical Stability, Electronic And Magnetic Properties Of Half- Heusler FeCrAs Alloy For Spintronics Application
}

\author{
${ }^{1 *}$ Chibueze T. C, ${ }^{2}$ Raji A. T and ${ }^{1}$ Ezema F. I \\ ${ }^{1}$ Department of Physics and Astronomy, University of Nigeria, Nsukka Enugu State, Nigeria. \\ ${ }^{2}$ Department of Physics, College of Science, Engineering and Technology (CSET), University of South Africa (UNISA), Florida \\ 1709, Roodepoort, Gauteng, South Africa.
}

\begin{abstract}
The search for functional materials in spintronic devices has become a major component of material research in recent times. The structural, elastic, mechanical, electronic and magnetic properties of half-Heusler FeCrAs alloy (HHFCA) have been examined adopting spinpolarized density functional theory calculations. Our result shows that the hexagonal structure is the high pressure phase of the FeCrAs alloy while the half-Heusler structure is the more stable phase at ambient pressure. Also, the HHFCA is mechanically stable and exhibits half-metallic ferromagnetism besides an indirect band gap in the minority spin channel. The total magnetic moment in one formula unit of the alloy is $1.00 \mu \mathrm{B}$, in agreement with the Slater-Pauling rule and the bulk of the magnetic moment contributed by the $\mathrm{Cr}$ atoms. Furthermore, high Curie temperature of $\sim 1000 \mathrm{~K}$ has been obtained for the HHFCA which suggests that it is a promising material for spintronic applications.
\end{abstract}

\section{ARTICLE HISTORY}

Received: $2^{\text {th }}$ July 2021

Revised: $20^{\text {th }}$ July 2021

Accepted: 30th Aug 2021

\section{KEYWORDS}

FeCrAs alloy

half-Heusler

mechanical stability

\section{INTRODUCTION}

Half-Heusler compounds (HHE) is a family of compounds possessing broad and enchanting qualities and applications. They have a cubic $\mathrm{Cl} b$ crystal structure in the space group F4́3m which is a three inter-penetrating face-centered-cubiclattices. In a normal HHE with the general formula $X Y Z$, the $X$ atom is a heavier transition element, $Y$ is a lighter transition element or a rare-earth and $Z$ is a late main-group element [1]. Also, there are three available atomic arrangements for the $X Y Z$ atoms often termed Type-1, Type-2 and Type-3. The atomic arrangements (respectively Wyckoff positions) are the following: Type-1 $(4 b(1 / 2,1 / 2,1 / 2), 4 c(1 / 4,1 / 4,1 / 4), 4 a(0,0,0))$; Type-2 $(4 b(1 / 2,1 / 2,1 / 2), 4 a(0,0,0), 4 c($ $1 / 4,1 / 4,1 / 4))$ and Type-3 $(4 c(1 / 4,1 / 4,1 / 4), 4 b(1 / 2,1 / 2,1 / 2), 4 a(0,0,0))$. Also, in the $\mathrm{Cl} b$ structure, $Y$ atoms constitute a rock-salt $(\mathrm{NaCl})$-like sublattice with the $Z$ atoms while the $X$ atoms on the other hand form a zinc-blende-like lattice with the $Z$ atoms.

The properties of the HHE phases significantly differ methodically along with the valence electron content (VEC), which is the periodic table prescribed valencies. These HHEs are characterized by large magnetic moments and high Curie temperatures [2-5] relative to the binary and associated phases [6]. In some HHEs, while the minority spin component displays an energy gap character at the Fermi level, the majority spin component exhibits metallic qualities as evidenced in the electronic structure. This phenomenon is called half-metallic ferromagnetic (HMF) character which culminates into $100 \%$ spin-polarized materials with high potential for technological applications in spintronics [5,7]. The inclusion of the spin accompaniment to the traditional semiconductor electronics has a lot of leverages such as, reduced electric power utilization, faster data processing speed, expanded integration densities and non-volatility [8].

FeCrAs alloy has been studied in two major phases: hexagonal and the HHE phase. Nylund et al. [9] synthesized hexagonal FeCrAs in the space group P6́m2 (No. 187). He et al. [10] investigated the crystal structure, elastic constants, thermodynamic and electronic properties of the novel hexagonal FeCrAs under high pressure using density functional theory. The electronic structure calculations of the hexagonal FeCrAs were performed with full-potential linearized augmented plane-wave (FPLAPW) method and the generalized gradient approximation (GGA) by Akrap et al. [11] where they assumed a paramagnetic ground state and non-interacting spins in these materials. Djelti et al. [12], Hao et al. [13] and Feng et al. [14] studied FeCrAs compounds in the half-Heusler structure with ferromagnetic ground state while Fujii et al. [15] obtained ferrimagnetic ground state for the half-Heusler FeCrAs (HHFCA) using FPLAPW method. The different ground states obtained using various approaches suggest that FeCrAs has different polymorphs. The results also suggest that the hexagonal phase is stabilized at high pressure while HHE phase is stablized at ambient and reduced pressure. 
In the present study, using the DFT approach, we examine the relative stability of hexagonal and HHE phases of FeCrAs alloy. Also, we determine originally the elastic constants and mechanical properties of FeCrAs in the HHE

\section{Computational Methodology}

An ab-initio DFT spin polarized calculations [16,17] have been performed using the corrected exchange correlation functional of Perdew-Burke-Ernzerhof (PBEsol) [18] which is a generalized gradient approximation (GGA). This functional have been shown to produce more accurately the equilibrium bulk and surface properties of close-packed solids. For these computations, plane-wave bais sets, as enabled in the Quantum-Espresso program package [19] was adopted. Electronic kinetic energy cutoff of 80 Ry with energy convergence benchmark of $10^{-8}$ Ry was utilized. Scalar relativistic norm-conserving pseudopotentials [20] were used to estimate the repulsion between ions and valence electrons. For the elements making up the HHE, the following are the valence electronic configurations: Fe: $4 s$, $4 d, 3 d$; $\mathrm{Cr}: 4 s, 4 p, 3 d$, and As: $4 s, 4 d$. Sampling of $k$-points in the Brillouin zone was done with $12 \times 12 \times 12$ grid with MonkhorstPack scheme [21] in the primitive unit cell of half-Heusler FeCrAs alloy presented in Figure 1. The Brillouin zone integration was carried out using the Marzari-Vanderbilt cold smearing scheme with a parameter of $0.01 \mathrm{Ry}$ and total energy convergence $\sim 1 \mathrm{mRy} / \mathrm{atom}$. The ionic geometry relaxation was relaxed to $\sim 0.1 \mathrm{mRy}$ for total energy and $1 \mathrm{mRy} / \mathrm{au}$ for the forces on the atoms. The equilibrium lattice constant of HHFCA was obtained from the total energy-lattice constants relation and fitting the calculated values to the Murnaghan equation of state [22]. The spin polarization $P$ is calculated using Eqn. (1) [23].

$$
P=\frac{D_{E_{F}}^{\uparrow}+D_{E_{F}}^{\downarrow}}{D_{E_{F}}^{\uparrow}-D_{E_{F}}^{\downarrow}} \times 100,
$$

where $D_{E_{F}}^{\uparrow}$ and $D_{E_{F}}^{\downarrow}$ are the density of states appearing at the Fermi level for spin-up and spin-down respectively.

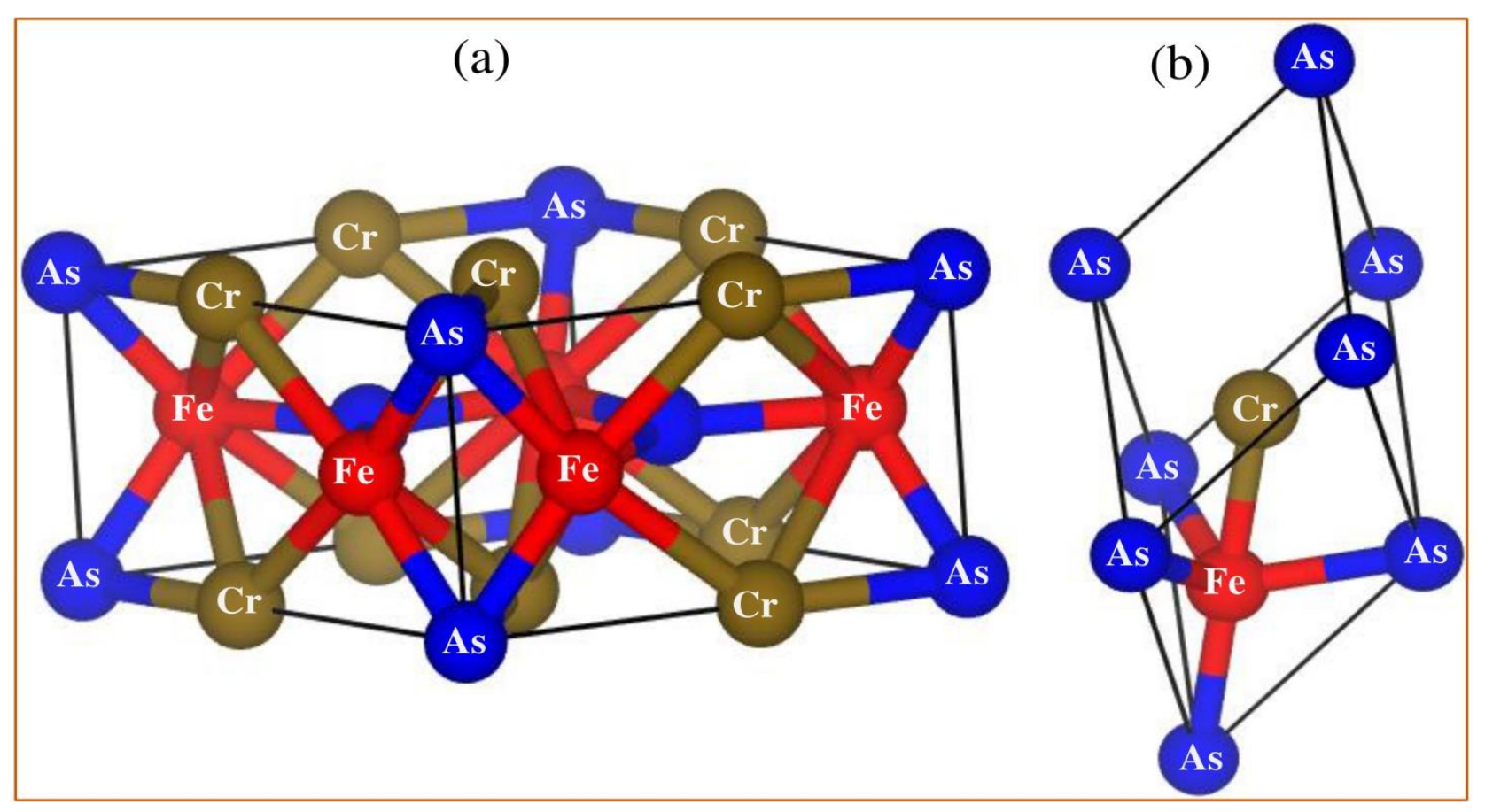

Figure 1: Crystal structure of FrCrAs alloy (a) hexagonal structure (b) half-Heusler structure

\section{RESULTS AND DISCUSSIONS}

\section{Structural and elastic properties}

We investigated the total energy of the two main structural phases of the FeCrAs alloy reported in the literature $[10,12,14,15]$, namely the hexagonal and half-Heusler phases. First, we performed total energy calculations for the Type1, Type- 2 and Type-3 phases of a half-Heusler structures. Our result shows that the Type-3 phase is the most stable phase as presented in Figure 2. We compared the structural stability of the hexagonal (Figure 1(a)) and the half-Heusler structure (Figure 1(b)). The magnetic ground state of the hexagonal polymorph is ferrimagnetic while that for the half-Heusler is ferromagnetic. Our result shows that the hexagonal polymorph is the high pressure phase. However, at the equilibrium lattice constant of the half-Heusler phase, both the hexagonal and half-Heusler phase have the same volume and total 
energy and so appears to be in equilibrium as presented in Figure 3. The equilibrium lattice constant of $5.59 \AA$ obtained in our calculation is in consonance with previous results presented in Table 1.

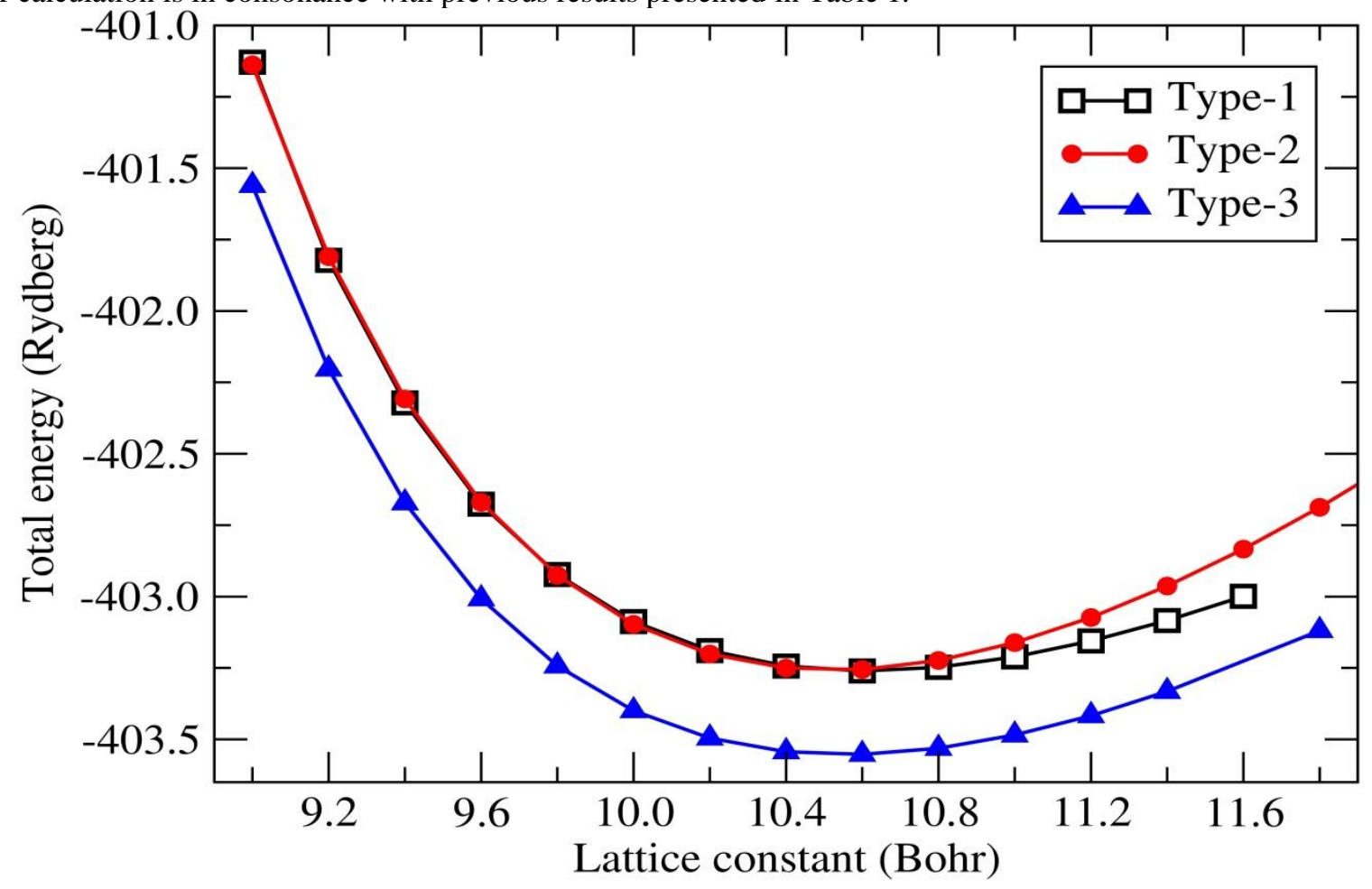

Figure 2: Total energy dependence on the lattice constant for Type-1, Type-2 and Type-3 half-Heusler phase of FeCrAs alloy.

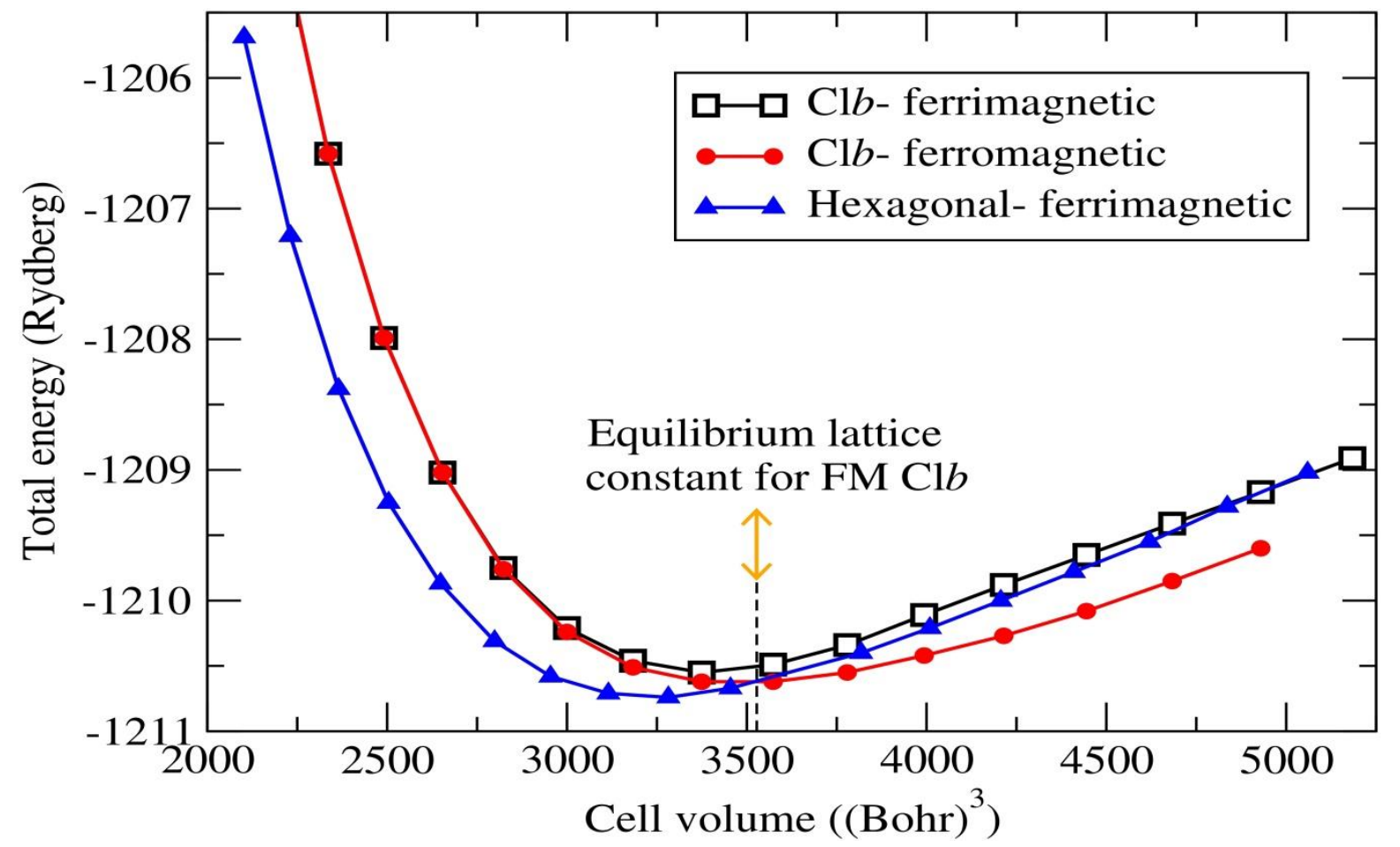

Figure 3: Total energy dependence on the unit cell volume of FeCrAs alloy in the half-Heusler phase (in both ferrimagnetic and ferromagnetic configuration) and hexagonal phase (in ferrimagnetic configuration).

Table 1: The lattice constant $(a)$, Young modulus $(Y)$, bulk modulus $(B)$, shear modulus $(G)$, Poission ratio $(v)$ and elastic constants (ie $C_{11}, C_{12}$ and $C_{44}$ ) of FeCrAs alloy in the half-Heusler ground state. 


\begin{tabular}{|c|c|c|c|c|c|c|c|c|c|}
\hline$a(\AA)$ & $Y(\mathrm{GPa})$ & $B(\mathrm{GPa})$ & $G(\mathrm{GPa})$ & $v$ & A & $\begin{array}{l}C_{11} \\
(\mathrm{GPa})\end{array}$ & $\begin{array}{l}C_{12} \\
(\mathrm{GPa})\end{array}$ & $\begin{array}{l}C_{44} \\
(\mathrm{GPa})\end{array}$ & Reference \\
\hline $\begin{array}{l}5.52^{\mathrm{a}} \\
5.48^{\mathrm{b}} \\
5.48^{\mathrm{c}} \\
5.51^{\mathrm{d}}\end{array}$ & & $161.598^{\mathrm{c}}$ & & & & & & & $\begin{array}{l}\text { Other } \\
\text { works }\end{array}$ \\
\hline 5.59 & 156.60 & 105.87 & 62.47 & 0.25 & 1.21 & 180.00 & 68.81 & 67.52 & This work \\
\hline
\end{tabular}

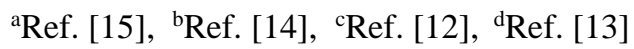

Three elastic constants comprising $\mathrm{C}_{11}, \mathrm{C}_{12}$ and $\mathrm{C}_{44}$ are vital to get the stability criteria of a cubic crystal. For a crystal subjected to strain by exerting stress presented as a stress tensor $\sigma_{i}$, for marginal magnitude of strain tensor $\epsilon_{j}$, the two tensors are related by [24]

$$
\sigma_{i}=\sum_{j=1}^{6} C_{i j} \epsilon_{j}
$$

The elastic tensor is obtained by subjecting the crystal lattice to finite distortions leading to the emergence of the elastic constants from the stress-strain Eqn.(2) [24]. The forces were minimized below $10^{-5} \mathrm{Ry} / \mathrm{au}$ for each strain using $16 \times 16 \times 16$ uniformly spaced Monkhorst-Pack grid. The lattice constants resulting from our calculations are presented in Table 1. The $C_{11}$ is $\sim 3$ times larger than $C_{44}$ in the HHFCA. This suggests that HHFCA is more resistant to deformation in one direction than to only shear strain. From Table 1, positive values of the cubic elastic constants satisfying the Born-Huang condition $[25,26]$ in Eqn. (3),

$$
\mathrm{C}_{11}-\mathrm{C}_{12}>0 ; \mathrm{C}_{11}+2 \mathrm{C}_{12}>0 ; \mathrm{C}_{44}>0,
$$

were obtained. This confirms that FeCrAs is mechanically stable in the HHE phase. Furthermore, bulk modulus $(B)$, Young's modulus $(Y)$ and shear modulus $(G)$ using the Voigt-Reuss-Hill (VRH) approximation [27,28].

We also estimated and presented Young modulus of HHFCA using the expressions: $Y=9 B G /(3 G+B)$ and Poisson ratio, $v=3 B-2 G / 2(3 B+G)[29]$. In general $v>0.26$ characterizes a material as ductile, else the compound is characterized as brittle. It is then obvious from Table 1 that half-Heusler FeCrAs alloy is brittle since we have obtained $v=0.25$. In addition, the elastic anisotropy was calculated using the expression $A=2 C_{44} /\left(C_{11}-C_{12}\right)$, where $A=1$ characterizes a material as isotropic while a $\mathrm{A} \neq 1$ characterizes it as anisotropic [30]. The degree of deviation from one (1) shows the height of elastic anisotropy possessed by the crystal material. Our result shows that HHFCA structure is anisotropic which usually serve to induce properties useful for spintronics [31].

\section{Electronic and magnetic Properties}

\section{Electronic properties}

The electronic properties of the HHFCA was studied using spin-polarized DFT calculations. The density of states from the atomic orbitals (PDOS) of the Fe, $\mathrm{Cr}$ and As are presented in Figure 4(a), (b) \& (c). The maximum of the valence band in the spin down component consists mainly of the Fe-3d orbitals while the maximum of the occupied states in the spin up component is basically that of the $\mathrm{Cr}$-3d orbitals. Also the bottom of the conduction band (which is unoccupied) in the spin down channel consists largely of the Cr-3d states. Figure 4 (d) is the total density of states (DOS) which shows that the spin up DOS is metallic while the spin down DOS displays electronic energy gap. It can be deduced from Figures 4(a-c) that the $\mathrm{Fe}-3 \mathrm{~d}$ and $\mathrm{Cr}-3 \mathrm{~d}$ orbitals is responsible for the electronic energy band gap in the spin down component. Figure 5 is the electronic band structure of the HHFCA. The spin up is metallic (Figure 5(a)) while the spin down electronic structure (Figure 5(b)) exhibits an indirect band gap with the top of the valence band at the symmetry point $\Gamma$ and the bottom of the valence band located at the symmetry points $\mathrm{X}$ in the Brillouin zone. The top of the valence band in the spin down band structure is triply degenerate while the bottom of the conduction band in the spin down component is doubly degenerate.

\section{Magnetic properties}

Our result shows that HHFCA exhibits ferromagnetic ground state contrary to the result of Fujii et al. [15] which reported ferrimagnetic ground state. The difference could be because due to the fact that we have used the corrected exchange correlation functional of Perdew et al. [18], commonly referred to as PBEsol which are known to give description of structures of solids. The total and local magnetic moments are presented in Table 2 . The bulk of the magnetic moment 
resides on the $\mathrm{Cr}$ atom as shown in the table. The magnetic moment on the $\mathrm{Cr}$ atom is antiparallel to that on the $\mathrm{Fe}$ and As atoms. The total magnetic moment $\mathrm{M}$ is $1.00 \mu_{\mathrm{B}}$ in consonance with the Slater-Pauling formula
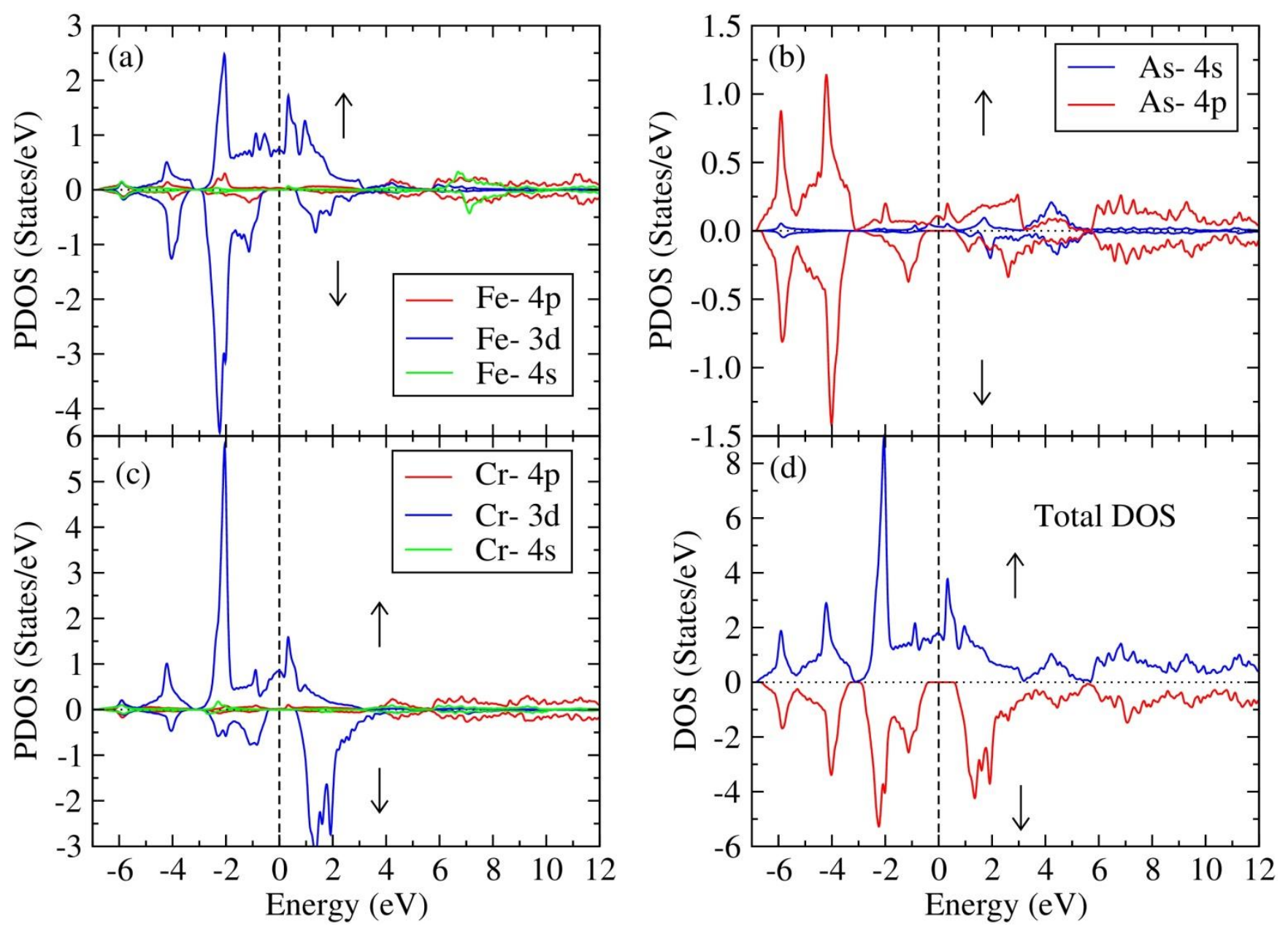

Figure 4: : Density of states projected on the atomic orbitals of (a) Fe (b) $\mathrm{Cr}$ (c) As in half-Heusler FeCrAS alloy (d) density of states (DOS) of half-Heusler KMnGe alloy for Type-3 structural phase. The blue line denotes the majority spin (spin up) DOS while the red line denote the minority spin (spin down)

Table 2: Magnetic properties of the half-Heusler FeCrAs alloy (HHFCA). The magnetic ground state (MGS), local magnetic moment on the $\mathrm{Fe}$ atom $\left(\mathrm{M}_{\mathrm{Fe}}\right)$, local magnetic moment on the $\mathrm{Cr}$ atom $\left(\mathrm{M}_{\mathrm{Cr}}\right)$, As atom local magnetic moment $\left(\mathrm{M}_{\mathrm{As}}\right)$, total magnetic moment $(\mathrm{M})$, spin polarization $(\mathrm{P})$, energy gap in the minority spin $\left(\mathrm{E}_{\mathrm{g}}\right)$ in the alloy.

\begin{tabular}{|l|l|l|l|l|l|l|l|l|l|}
\hline MGS & $\begin{array}{l}\mathrm{M}_{\mathrm{Fe}} \\
\left(\mu_{\mathrm{B}}\right)\end{array}$ & $\begin{array}{l}\mathrm{M}_{\mathrm{Cr}} \\
\left(\mu_{\mathrm{B}}\right)\end{array}$ & $\begin{array}{l}\mathrm{M}_{\mathrm{As}} \\
\left(\mu_{\mathrm{B}}\right)\end{array}$ & $\begin{array}{l}\mathrm{M} \\
\left(\mu_{\mathrm{B}}\right)\end{array}$ & $\mathrm{P}(\%)$ & $T_{C}(\mathrm{~K})$ & $E_{g}(\mathrm{eV})$ & $E_{g}$ Type & Reference \\
\hline FM & -1.36 & 2.43 & -0.07 & 1.00 & 100 & 1004.14 & 1.16 & Indirect & This work \\
\hline FiM & -0.73 & 1.67 & & 1.00 & 100 & & & & Ref.[15] \\
\hline & & & & & 100 & & 1.28 & Indirect & Ref.[12] \\
\hline FM & -0.88 & 2.03 & -0.15 & 1.00 & 100 & & 0.58 & Direct & Ref.[13] \\
\hline & -0.60 & 1.80 & & 1.00 & 100 & & 0.88 & & Ref.[14] \\
\hline
\end{tabular}



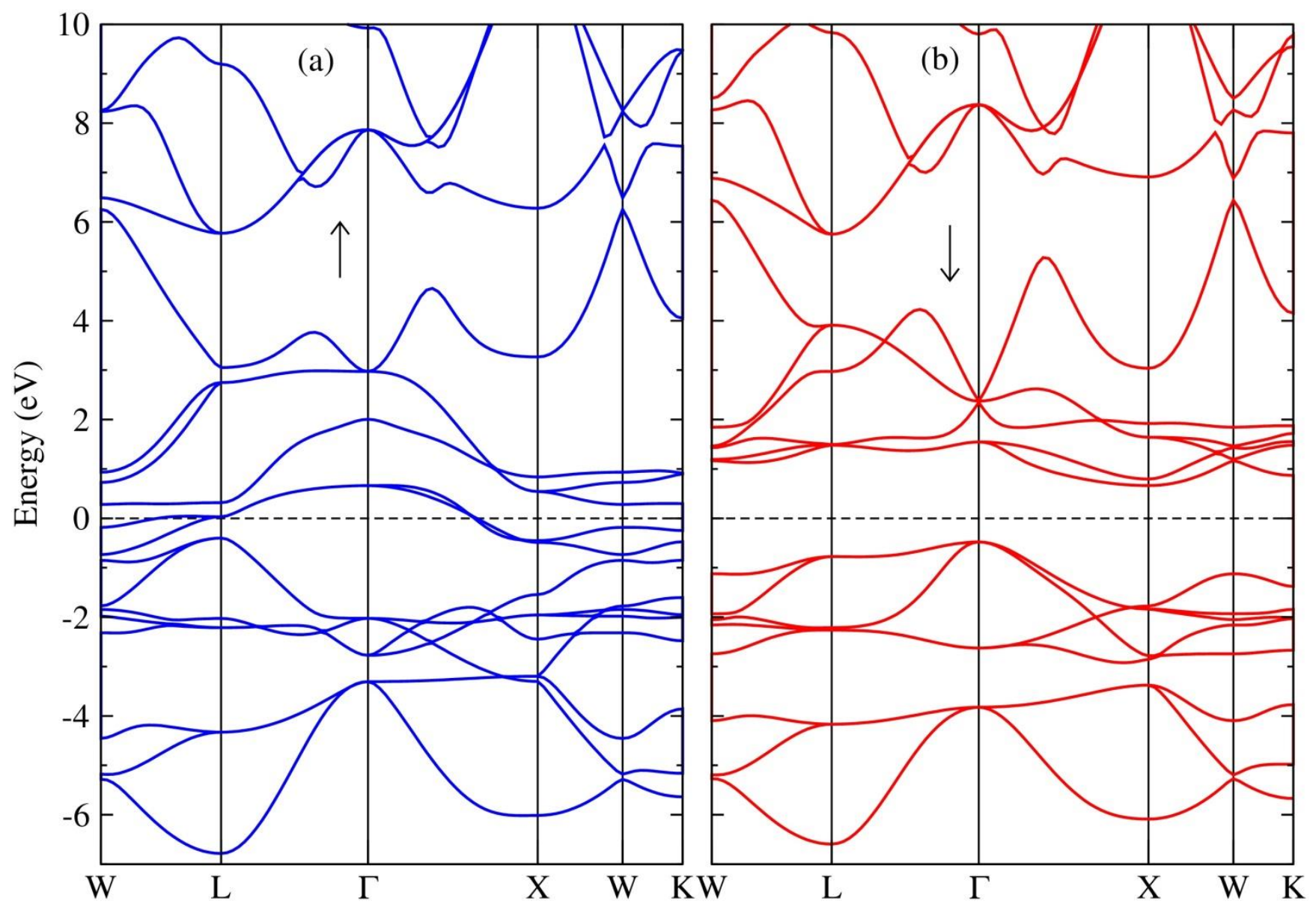

Figure 5: Electronic band structure of half-Heusler FeCrAs alloy for Type-3 structural phase. (a) majority spin states (spin up) (b) Minority spin states (spin down). The dashed horizontal line is the Fermi energy.

The spin polarization was calculated using Eqn. (1). We use the mean field approximation of Heisenberg model to compute the Curie temperature $\left(\mathrm{T}_{\mathrm{C}}\right)[32], T_{\mathrm{C}}=2\left(\mathrm{E}_{\mathrm{AFM}}-\mathrm{E}_{\mathrm{FM}}\right) / 3 \mathrm{~K}_{\mathrm{B}}$,

where

$\mathrm{E}_{\mathrm{AFM}}, \mathrm{E}_{\mathrm{FM}}$ and $\mathrm{K}_{\mathrm{B}}$ are respectively the total energies of the antiferromagnetic and ferromagnetic states, as well as Boltzmann constant. Our calculated $T_{\mathrm{C}} \sim 1000 \mathrm{~K}$ of half-Heusler FeCrAs is very high thus making this alloy an excellent material for spintronic applications.

\section{CONCLUSIONS}

The structural, elastic, mechanical, electronic and magnetic properties of the HHFCA have been studied using the spinpolarized DFT calculations. Our result showed that hexagonal phase is the stable phase at high pressure while the HHE phase is the more stable at ambient pressure. Our result show that Type-3 is the HHE structural ground state with ferromagnetic configuration. The HHFCA is mechanically stable, brittle and anisotropic. Electronic properties showed that HHFCA exhibits half-metallic ferromagnetism with an indirect band gap of $1.16 \mathrm{eV}$ in the minority spin channel and $100 \%$ spin polarization around the Fermi level. The result of the magnetic properties showed that the total magnetic moment contained in one formula unit is $1.00 \mu_{\mathrm{B}}$ in consonance with the Slater-Pauling prediction. The bulk of the magnetic moment resides on the $\mathrm{Cr}$ atoms. High Curie temperature was obtained for HHFCA which suggests that the compound is promising for spintronic applications.

\section{ACKNOWLEDGEMENT}

Center for High Performance Computing, Cape Town, South Africa is appreciated for providing their resources. 


\section{REFERENCES}

[1] Chibueze TC, Raji AT, Okoye CM. First principles study of the effects of doping with sp elements (As, Sb, Bi) and pressure on the properties of half-Heusler AuMnSn. Chemical Physics 2020; 530: 110635.https://doi.org/10.1016/j.chemphys.2019.110635

[2] Offernes L, Torgersen AN, Kjekshus A. The ternary system Au-Mn-Sn. Journal of Alloys and Compounds 2000; 307(1-2): 174178.https://doi.org/10.1016/S0925-8388(00)00885-9

[3] Galanakis I, Dederichs PH, Papanikolaou NJ. Origin and properties of the gap in the half-ferromagnetic Heusler alloys. Physical Review B, 2002; 66(13): 134428. https://doi.org/10.1103/PhysRevB.66.134428

[4] Chibueze TC, Ekuma CE, Raji AT, Rai DP, Okoye CMI. Ferromagnetic half-metallicity in half-Heusler AuMnSn: Te Alloy. Materials Research Express 2020; 7(7): 076519.https://doi.org/10.1088/2053-1591/ab88ff

[5] Ležaić M, Galanakis I, Bihlmayer G, Blügel S. Structural and magnetic properties of the (001) and (111) surfaces of the half-metal NiMnSb. Journal of Physics: Condensed Matter 2005; 17(21): 3121. https://doi.org/10.1088/0953-8984/17/21/008

[6] Reshak AH. Thermoelectric properties of the spin-polarized half-metallic ferromagnetic CsTe and RbSe compounds. RSC advances 2016; 6(100): 98197-98207. https://doi.org/10.1039/C6RA22758A

[7] Chibueze TC, Ekuma CE, Raji AT, Ezema FI, Okoye CMI. Tetragonal and uniaxial strains in pristine and doped half-Heusler AuMnSn alloy. Journal of Alloys and Compounds 2020; 848: 156186. https://doi.org/10.1016/j.jallcom.2020.156186.

[8] Prinz GA. Magnetoelectronics applications. Journal of Magnetism and Magnetic Materials 1999; 200(1): 57-68. https://doi.org/10.1016/S0304-8853(99)00335-2

[9] Nylund MA, Roger MM, Sénateur JP, Fruchart R. Evolution structurale des phosphures, arséniures et arséniophosphures M2P, M2As et M2 (P1-xAsx). Journal of Solid State Chemistry 1972; 4(1): 115-22. https://doi.org/10.1016/0022-4596(72)90139-9

[10] He DC, Peng Y, He YW. Ab Initio Studies on Structural, Elastic, Thermodynamic and Electronic Properties of FeCrAs under Pressures. Acta Physica Polonica A, 2015; 127(6): 1637-1644. http://dx.doi.org/10.12693/APhysPolA.127.1637

[11] Akrap A, Dai YM, Wu W, Julian SR, Homes CC. Optical properties and electronic structure of the nonmetallic metal FeCrAs. Physical Review B, 2014; 89(12): 125115. https://doi.org/10.1103/PhysRevB.89.125115

[12] Djelti R, Besbes A, Bestani B. TB-mBJ calculations of optical and thermoelectric properties of half-Heusler FeCrAs alloy. Optical and Quantum Electronics 2020; 52(9): 1-0. https://doi.org/10.1007/s11082-020-02527-X

[13] Hao Z, Liu R, Fan Y, Wang L. First-principles calculations of a new half-metallic Heusler alloy FeCrAs. Journal of Alloys and Compounds 2020; 820: 153118. https://doi.org/10.1016/j.jallcom.2019.153118

[14] Feng L, Liu EK, Zhang WX, Wang WH, Wu GH. First-principles investigation of half-metallic ferromagnetism of half-Heusler compounds XYZ. Journal of magnetism and magnetic materials 2014; 351: 92-97. https://doi.org/10.1016/j.jmmm.2013.09.054

[15] Fujii S, Ishida S, Asano S. High Spin Polarization of Ferrimagnetic Half-Heusler Compounds FeCrZ and MnYZ (Y= Mn, Cr; Z= IIIb, IVb, Vb Elements). Journal of the Physical Society of Japan 2010; 79(12): 124702. https://doi.org/10.1143/JPSJ.79.124702

[16] Hohenberg P, Kohn, W. Inhomogeneous electron gas. Physical Review 1964, 136, B864 B871.https://doi.org/10.1103/PhysRev.136.B864

[17] Kohn W, Sham, LJ. Self-consistent equations including exchange and correlation effects. Physical Review 1965; 140: A1133-A1138. https://doi.org/10.1103/PhysRev.140.A1133

[18] Perdew, J. P., Ruzsinszky, A., Csonka, G. I., Vydrov, O. A., Scuseria, G. E., Constantin, L. A. and Burke, K. (2008). Restoring the density-gradient expansion for exchange in solids and surfaces. Physical review letters, 100(13), 136406. https://doi.org/10.1103/PhysRevLett.100.136406

[19] Giannozzi P, Baroni S, Bonini N, Calandra M, Car R, Cavazzoni C, Ceresoli D, Chiarotti GL, Cococcioni M, Dabo I, Corso AD, de Gironcoli S, Fabris S, Fratesi G, Gebauer R, Gerstmann U, Gougoussis C, Kokalj A, Lazzeri M, Martin-Samos L, Marzari N, Mauri F, Mazzarello R, Paolini S, Pasquarello A, Paulatto L, Sbraccia C, Scandolo S, Sclauzero G, Seitsonen AP, Smogunov A, Umari P, Wentzcovitch RM. QUANTUM ESPRESSO: a modular and open-source software project for quantum simulations of materials. Journal of Physics: Condensed Matter 2009; 21: 395502.https://doi.org/10.1088/0953-8984/21/39/395502

[20] Hamann DR, Schlüter M, Chiang C. Norm-conserving pseudopotentials. Physical Review Letters, 43(20), 1494. https://doi.org/10.1103/PhysRevLett.43.1494

[21] Monkhorst HJ, Pack JD. Special points for Brillouin-zone integrations, Physical Review B, 1976; 13: 5188-5192. https://doi.org/10.1103/PhysRevB.13.5188

[22] Murnaghan FD. The compressibility of media under extreme pressures. Proceedings of the National Academy of Sciences 1944; 30(9): 244-247. https://dx.doi.org/10.1073\%2Fpnas.30.9.244

[23] Chibueze TC, Raji AT, Okoye CMI. Intrinsic point defects in half-Heusler AuMnSn. Journal of Physics and Chemistry of Solids 2020; 139: 109328. https://doi.org/10.1016/j.jpcs.2019.109328

[24] Nielsen OH, Martin RM. Quantum-mechanical theory of stress and force. Physical Review B, 1985; 32(6): 3780- 
3791. https://doi.org/10.1103/PhysRevB.32.3780

[25] Born M. On the stability of crystal lattices I. Mathematical Proceedings of the Cambridge Philosophical Society 1940; 36(2): 160-172. https://doi.org/10.1017/S0305004100017138

[26] Mouhat F, Coudert FX. Necessary and sufficient elastic stability conditions in various crystal systems. Physical Review B, 2014; 90(22): 224104. https://doi.org/10.1103/PhysRevB.90.224104

[27] Hill R. The elastic behaviour of a crystalline aggregate. Proceedings of the Physical Society 1952; 65(5): 349354. https://doi.org/10.1088/0370-1298/65/5/307

[28] Weck PF, Kim E. Assessing Hubbard-corrected AM05+U and PBEsol+U density functionals for strongly correlated oxides $\mathrm{CeO} 2$ and Ce2O3. Physical Chemistry Chemical Physics 2016; 18(38): 26816-26826. https://doi.org/10.1039/C6CP05479J

[29] Wu DH, Wang HC, Wei LT, Pan RK, Tang BY. First-principles study of structural stability and elastic properties of $\mathrm{MgPd} 3$ and its hydride. Journal of Magnesium and Alloys 2014; 2(2): 165 - 174. https://doi.org/10.1016/j.jma.2014.06.001

[30] Haines J, Léger JM, Bocquillon G. Synthesis and design of superhard materials. Annual Review of Materials Research 2001; 31(1): 1-23. https://doi.org/10.1146/annurev.matsci.31.1.1

[31] Li X, Yang J. First-principles design of spintronics materials. National Science Review 2016; 3(3): $365-381$. https://doi.org/10.1093/nsr/nww026

[32] Blundell S. Magnetism in condensed matter. New York: Oxford University Press; 2003. 